\title{
The thermal-viscous disk instability model in the AGN context
}

\author{
Jean-Marie Hameury, ${ }^{1}$ Jean-Pierre Lasota ${ }^{2}$ and Maxime Viallet ${ }^{1}$ \\ ${ }^{1}$ Observatoire de Strasbourg, CNRS/Université Louis Pasteur, \\ 11 rue de l'Université, 67000 Strasbourg, France \\ ${ }^{2}$ Institut d'Astrophysique de Paris, CNRS/Université Pierre et Maris Curie, \\ 98bis Bd. Arago, 75015 Paris, France \\ email: hameury@astro.u-strasbg.fr; viallet@astro.u-strasbg.fr
}

\begin{abstract}
Accretion disks in AGN should be subject to the same disk instability responsible for dwarf novae outbursts and soft X-ray transients in cataclysmic variables (CVs) and LMXBs. It has been suggested that this thermal/viscous instability can account for long term variability of AGNs. We analyze here the application of the DIM to the AGN case, using our adaptive grid numerical code developed in the context of CVs, enabling us to fully resolve the disk radial structure. We show that in AGNs, the width of heating and cooling fronts is so small that they cannot be resolved by standard codes, and that they propagate on time scales much shorter than the viscous time. As a result, transition fronts propagate back and forth in the disk, leading only to small luminosity variations. Truncation of the inner part of the disk by e.g. an ADAF will not alter this result, but enables the presence of quiescent states.
\end{abstract}

Keywords. accretion, accretion disks - instabilities - galaxies: active - stars: dwarf novae

\section{Introduction}

Accretion disks are found in a large variety of astronomical objects, from young stars to active galactic nuclei. Among these, close binaries deserve special attention, because they are nearby, and vary on short timescales that enable time-dependent studies of their light curve. In particular, a number of these systems show large outbursts, as for example dwarf novae (DN), which are a subclass of cataclysmic variables. These outbursts are believed to be due to a thermal-viscous accretion disk instability which arises when the disk temperature becomes of order of $10^{4} \mathrm{~K}$, enough for hydrogen to become partially ionized and opacities to depend strongly on temperature (Lasota 2001). Similarly, soft X-ray transients, a subclass of low-mass X-ray binaries, also show outbursts, their amplitude being, in general, larger and the time scales longer than for dwarf novae. The ionization instability of the accretion disk is also thought to be the cause of the outbursts, the difference with DNe being due to larger masses of the compact objects (and thus to deeper gravitational potential wells) and to the effect of illumination of the disk, much more important in the case of X-ray binaries (Dubus et al. 2001).

It was realized long ago (Lin \& Shields 1986) that the same instability could be present in accretion disks in AGNs; it was found that, at radii $\sim 10^{15-16} \mathrm{~cm}$, where the effective temperature is indeed of a few thousand degrees, the disk should be unstable. For the parameters of AGNs, the implied timescales are of order of $10^{4}-10^{7} \mathrm{yr}$, making impossible the direct observation of the instability in a particular object, but implying that in many systems the disk is not in viscous equilibrium and that many AGNs are in a quiescent state. It was also immediately realized that, as in DNe, the character of putative AGN outbursts strongly depends on assumptions one makes about the disk viscosity (Mineshige 

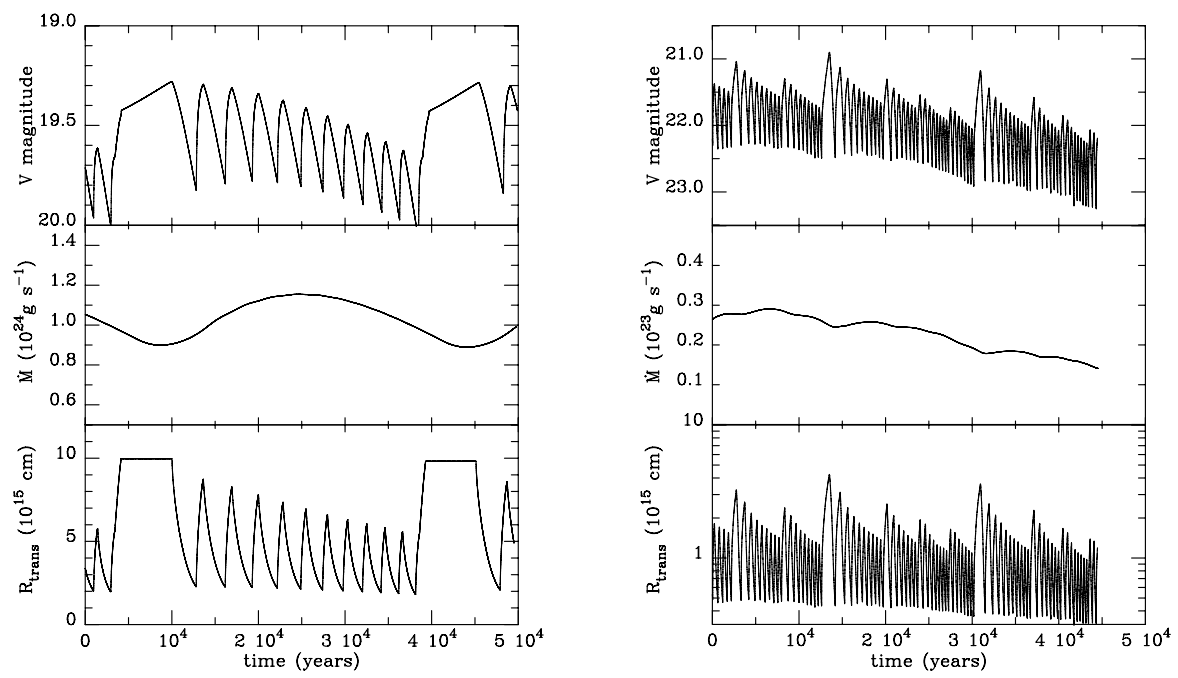

Figure 1. Left: Time evolution of an accretion disk with the following parameters: black hole mass: $10^{8} \mathrm{M}_{\odot}$, inner and outer radius: $10^{14}$ and $10^{16} \mathrm{~cm}$ respectively, mean mass transfer rate: $10^{24} \mathrm{~g} \mathrm{~s}^{-1}$. Top panel: visual magnitude, intermediate panel: accretion rate onto the black hole, lower panel: radius at which the transition between the hot and cold regimes takes place. Right: same parameters except that $\dot{M}=210^{22} \mathrm{~g} \mathrm{~s}^{-1}$.

\& Shields 1990). However, while in the case of DNe one is guided in fixing the viscosity prescription by the observed outburst properties, in the case of AGN it is not even clear that outbursts are present as the variability of these objects could be due just to masssupply variations. (In many cases it is not even clear that a disc is present.) This state of affairs gave rise to various, more or less arbitrary, prescriptions of how viscosity varies (or not) with the state of the accretion flow (Mineshige \& Shields 1990; Menou \& Quataert 2001; Janiuk et al. 2004). In addition, results of numerical calculations of AGN outbursts were marred by the insufficient resolution of grids used. As showed by Hameury et al. (1998) low grid resolution often leads to unreliable, unphysical, results.

We revisit here the application of the disk instability model in the context of AGNs, using the code we have developed in the context of dwarf novae and soft X-ray transients.

\section{Disk evolution}

We solve the standard equations for mass and angular momentum conservation in a geometrically thin accretion disk. The heat conservation involves the heating and cooling rates of the unsteady disk; in other words, one must know the effective temperature $T_{\text {eff }}$ as a function of radius $r$, surface density $\Sigma$ and central temperature $T_{\mathrm{c}}$. We use the standard approximation that $T_{\text {eff }}$ does not depend on any other parameter than $r, \Sigma$ and $T_{\mathrm{c}}$, and is thus found by solving for the vertical structure of the disk in steady state, the value of the Shakura-Sunyaev parameter $\alpha$ being not constrained.

We calculate a grid of vertical structures in the same way as in Hameury et al. (1998). We do not include self gravity, which can be important at large distances from the black hole, typically $1-210^{16} \mathrm{~cm}$ for a $10^{8} \mathrm{M}_{\odot}$ black hole (Cannizzo 1992; Cannizzo \& Reiff 1992). When self gravity dominates, the disk becomes gravitationally unstable, which may lead to fragmentation if the cooling time is short enough, or significantly change the angular momentum transport by introducing non local terms (see e.g. Lin \& Pringle 1987; Balbus \& Papaloizou 1999). In any case, the thermal-viscous instability model can 


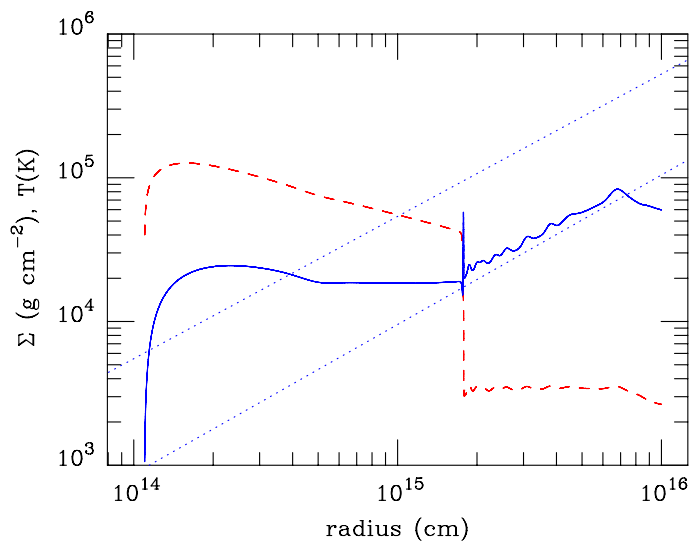

Figure 2. Radial structure of a disk. The blue solid line represents the surface density, the red dashed one the central temperature. The dotted lines are the critical $\Sigma_{\min }$ and $\Sigma_{\max }$.

no longer apply, and we always make sure that, in our calculations, self gravity is never important.

In the standard DN model, it is assumed that the $\alpha$-parameter changes rapidly when the disk temperature reaches the ionization instability; this is required for the amplitude of the modelled instability to be comparable to the observed one. It has been suggested that $\alpha$ might remain constant in AGN disks (Gammie \& Menou 1998; Menou \& Quataert 2001); however, this proposal is based on numerical simulations which are far from providing us with a complete treatment of the turbulent dynamics of disks (Balbus, private communication), and we use here the standard DN model for viscosity.

Fig. 1 shows the evolution of a disk around a $10^{8} \mathrm{M}_{\odot}$ black hole, for two different values of the mass transfer rate: $1 \%$ and $.01 \%$ of the Eddington limit, assuming a $10 \%$ efficiency. In both cases, $\alpha_{\text {hot }}=5 \alpha_{\text {cold }}=0.2$, and the inner and outer disk radius are $10^{14}$ and $10^{16} \mathrm{~cm}$ respectively. The disk is indeed unstable; however, this instability does not result in large outbursts, but rather in rapid oscillations reminiscent of what is found in dwarf novae when $\alpha$ is assumed to be constant.

The basic reason for this is that transition fronts propagates at approximately $\alpha$ times the sound speed (Menou et al. 1999), i.e. on a time scale

$$
t_{\text {front }}=\frac{r}{\alpha c_{\mathrm{s}}}=\frac{r}{h} t_{\mathrm{th}}
$$

where $t_{\mathrm{th}}$ is the thermal time scale. $t_{\text {front }}$ is shorter than the viscous time $t_{\mathrm{visc}}=(r / h)^{2} t_{\mathrm{th}}$ by a factor $r / h$, i.e. by several orders of magnitude. The cooling front therefore propagates so rapidly that the surface density at smaller radii does not change; to a first approximation, it cannot propagate in regions where $\Sigma>\Sigma_{\max }\left(\alpha_{\text {cold }}\right)$, the maximum surface density for a cold stable disk. In the CV case, $t_{\text {front }}$ is shorter than $t_{\text {visc }}$, but not by such a large amount and strong gradients in the disk make the effective viscous time comparable to the the front propagation time. When $\Sigma_{\max }$ is reached, the cooling front is reflected into a heating front, which propagates outwards until the outer disk edge is reached, or $\Sigma$ is less than $\Sigma_{\min }\left(\alpha_{\text {hot }}\right)$ (the minimum surface density of a hot stable disk). The radial profile of the disk is shown in Fig. 2, in the case where $\dot{M}$ is low. There are two stable inner and outer regions which are clearly seen, where the disk is always hot (inner zone) or cold (outermost regions). The intermediate, unstable zone is divided in two regions: an inner unstable part, and an outer marginally stable one, where $\Sigma=\Sigma_{\min }(r)$, resulting from the successive passage of heating fronts that die at radii decreasing with time; a leftover of the death of these fronts is a little wiggle in $\Sigma$ that get smoothed with time as a result of diffusion, or when a heat front is able to cross a large distance. Note 
also the spike in the unstable region, which carries a small amount of mass that will cause the small wiggles in the marginally stable region.

The minimum radius reached by the cooling front can de determined by noting that the front propagates down to a point where $\Sigma=\Sigma_{\max }\left(\alpha_{\text {cold }}\right)$, and that the innermost parts of the disk are in quasi viscous equilibrium, which means that the surface density is determined by the accretion rate, almost constant in this hot inner region. The minimum radius reached by cooling fronts can then be written as:

$$
r=1.7 \times 10^{15}\left(\frac{\dot{M}}{10^{23} \mathrm{~g} \mathrm{~s}^{-1}} M_{8} \frac{\alpha_{\text {cold }}}{\alpha_{\text {hot }}} f\right)^{0.4} \mathrm{~cm}
$$

where $f=1-\left(r_{\text {in }} / r\right)^{1 / 2} \sim 1$; this shows that, unless the average accretion rate is extremely small, a cold quiescent state can never be reached.

\section{Discussion}

We have shown that the straightforward application of the disk instability model to AGN does lot lead to large amplitude outbursts, but to some sort of flickering. This is because in AGN, the Mach number is so large that the propagation time of heating and cooling fronts is much less than the viscous time. Evaporation of the innermost parts due e.g. to the formation of an ADAF will change this conclusion, as low states will occur, as shown by Eq. 2.2, since if the disk is truncated at a few Schwarzschild radii, cooling fronts can reach the inner disk edge even for relatively high $\dot{M}$; the flickering behaviour in high states will remain though. The amplitude of the outbursts, as well as the maximum luminosity reached remain to be determined. Irradiation of the disk by X-rays might also affect the outcome of the instability; Burderi et al. (1998) showed that irradiation does not prevent the instability, and, because it does not affect the propagation time of the front nor the viscous time, it in unlikely that it can have a major effect.

\section{References}

Balbus, S. A. \& Papaloizou, J. C. B. 1999, ApJ, 376, 214

Burderi, L., King, A. R. \& Szuszkiewicz, E. 1998, ApJ, 509, 85

Cannizzo, J. K. 1992, ApJ, 385, 94

Cannizzo, J. K. \& Reiff, C. M. 1992, ApJ, 385, 87

Dubus, G., Hameury, J.-M. \& Lasota, J.-P. 2001, A\&A, 373, 251

Gammie, C. F. \& Menou, K. 1998, ApJ, 492, L75

Hameury, J.-M., Menou, K., Dubus, G., Lasota, J.-P. \& Huré, J. M. 1998, MNRAS, 298, 1048

Janiuk, A., Czerny, B., Siemiginowska, A. \& Szczerba, R. 2004, ApJ, 602, 595

Lasota, J.-P. 2001, New Astron. Revs, 45, 449

Lin, D. N. C. \& Pringle, J. E. 1986, MNRAS, 225, 607

Lin, D. N. C. \& Shields, G. 1986, ApJ, 305, 28

Menou, K. \& Quataert, E. 2001, ApJ, 552, 204

Menou, K., Hameury, J.-M. \& Stehle, R. 1999, MNRAS, 305, 79

Mineshige, S. \& Shields, G. A. 1990, ApJ, 351, 47

SuzY Collin: How do you avoid to enter in the ADAF/ADIOS/... regime at mass-rate that is typically below $1 / 100$ of the Eddington limit?

JEAN-MARIE HAMEURY: It is not known at present what is the minimum accretion rate for the standard Shakura-Sunayev solution to apply; the 1/100 limit you quote refers to a maximum value for ADAF to be possible. In any case, the formation of an ADAF/ADIOS etc. will result in truncation of the inner disc, and this is included in the calculations I have shown. 